\title{
LA SOSTENIBILIDAD EN EL SISTEMA MODA
}

Sustainability in the Fashion system

\author{
Angela Ramos López \\ aramosl@poligran.edu.co \\ Janeth Puentes Bedoya \\ jpuentesb@poligran.edu.co \\ Bárbara Elena Díaz Barrios \\ badiazba@poligran.edu.co \\ Julieth Vanesa Ávila Gamboa \\ juavilag@poligran.edu.co \\ Laura Sofía Rueda Cascante \\ laruedac@poligran.edu.co \\ Manuela Valentina Caicedo \\ macaicedo11@poligran.edu.co \\ Angie Esperanza León Pardo \\ anleonpa@poligran.edu.co \\ Cindy María Garagoa Marín \\ cigaragoa@poligran.edu.co \\ Erika Juliette Guevara \\ erguevara1@poligran.edu.co
}

Institución Universitaria Politécnico Grancolombiano FIDI - Escuela de Diseño - Programa Diseño de Modas Semillero de Investigación: Moda, Comunicación y Sostenibilidad

Bogotá, Colombia

\section{RESUMEN}

La sostenibilidad en el diseño de moda nace a partir de la necesidad de análisis y visibilización de los procesos construidos a partir de acuerdos sociales innovadores y acciones colectivas, que han permitido generar un pensamiento reflexivo y desarrollar el sistema moda; igualmente, como se han construido desde una manera desmedida al afectar el entorno sociocultural, ambiental y económico, el negocio de la industria de la moda debe transformarse y reinventarse para reconciliarse con la tierra y con los seres humanos en general. Por consiguiente, este proyecto en curso tiene como propósito crear y generar conciencia a partir de la inquietud que surge desde la academia hasta la ética de la labor del diseñador de moda cuya labor se enfoca en varios frentes como comunicador, educador y facilitador.

PALABRAS CLAVE:

Diseño, moda sostenible, sistema moda

Cite este artículo como: Ramos, A., Puentes, J., Díaz, B., Ávila, J., Rueda, L., ...\& Guevara, E. (2020). La sostenibilidad en el sistema moda. 


\section{INTRODUCCIÓN}

El concepto de sostenibilidad dentro de la industria de la moda tiene que ver con el nuevo significado evolutivo de la misma industria; tiene que ver con toda aquella ropa que se fabrica y no compromete los recursos de las futuras generaciones. En efecto, que tenga como principio fundamental el respeto, tanto con el medio ambiente como a nivel social, en este caso con las personas que hacen parte del proceso creador del diseño y de su producción. De acuerdo con la Ethical Fashion Forum (https://the.ethicalfashionforum.com/about-1), la ética de la moda representa un acercamiento al diseño, a su búsqueda y a una mejor manufactura de las prendas, la cual beneficie enormemente a la gente y a las comunidades para minimizar el impacto ambiental.

Igualmente, para la Ethical Fashion Forum, el significado va más allá de no hacer daño, sino que representa un agrupamiento que trabaja por tomar un rol activista para reducir la pobreza, que genere sustento, minimizando y contrarrestando las preocupaciones ambientales. Es así como la palabra sostenibilidad no solo se basa en lo ambiental, sino que también la industria de la moda debe asumir la parte social de la siguiente manera: incrementar la posibilidad y el bienestar de la gente y de las comunidades, quienes están detrás de la industria de la moda, ya que depende de los trabajadores que se encuentran apoyando con sus funciones diarias a la industria como tal. En otras palabras, la moda refleja las transformaciones de una sociedad, de un periodo en la historia; es un vehículo por el cual se le propone al individuo unos determinados valores que dan como resultado una identidad cultural y social (San Martín, 2009), que se construye en un vínculo dado entre las relaciones de producción y simbólicas (Monneyron, 2006), siendo de esta manera pertinente el entender a la sostenibilidad como una vía de desarrollo desde la perspectiva productiva, pero también desde la vinculación afectiva y justa.

Por otro lado, existe una gran preocupación en los gremios como Common Objective, Fashion Revolution, entre otros, y a su vez, desde la mirada de los pequeños grupos de diseñadores emergentes con respecto al Fast Fashion, el cual ha sido un detonante en el exceso de producción de prendas y que ha sido una de las causantes del desproporcionado manejo de la industria de la moda en sí, ya que el interés que tienen las marcas afiliadas a este sistema de la moda rápida es netamente económico a un nivel desmedido. El Fast Fashion se consolida a partir de la penetración en el mercado propio, en la globalización, la integración vertical, la diversificación y los precios asequibles; gracias a esos factores es que ha ganado un gran terreno y se ha convertido en un gran muro que impide el desarrollo de la creatividad y producción conscientes; en efecto, es una parte del sistema moda que no ha dejado respirar a la naturaleza y al entorno en su conjunto, ya que lleva consigo una serie de consecuencias nefastas para los humanos.

Es por esta razón por la que la moda no solamente debe relacionarse con el objetivo de proponer unos valores determinados, sino que también es un sistema con el cual se desarrollan procesos de sensibilización que pueden generar y recrear experiencias sanadoras, en donde los objetos que la conforman son una manifestación física de cómo se interpreta el mundo y cómo los seres humanos le damos forma para reflejar quiénes somos como individuos (Fletcher \& Grose, 2012), desde la experiencia con las cosas, es decir, las relaciones materiales infinitas que se unen en los sistemas socioculturales, económicos y la naturaleza (Fletcher \& Grose, 2012), siendo esto el resultado de la emergencia de continuas iniciativas artísticas, en particular en el campo de la sostenibilidad, que se inscriben más allá de la construcción de la tradicional obra de arte, generando proyectos abiertos en los cuales participan los ciudadanos del común, y se van entretejiendo relaciones, espacios, sueños e ideas, que además de mantener viva la voz de los individuos tienen un efecto liberador y fortalecen los lazos sociales.

Este proyecto busca generar un espacio a partir de la temática de la sostenibilidad desde la perspectiva de la industria de la moda (diseñadores y/o empresarios y consumidores), en el mundo y en Colombia, con el fin de concientizar y sensibilizar a la sociedad a cambiar hábitos de consumo y producción mediante la divulgación y aplicación de nuevas maneras de reutilizar, transformar y reciclar prendas, para así generar estrategias de adaptabilidad y evolución. 
Del mismo modo, la finalidad es entrever las problemáticas que existen dentro del sistema moda y su industria a partir de la indagación de aquellas problemáticas, que tanto el empresario (diseñador-productor textil) como el consumidor proyectan desde la sostenibilidad, talleres de sensibilización y reconstrucción de prendas para proponer una producción amigable con la naturaleza, ya que la industria de la moda impacta a tantos, y como tal, tiene el potencial de mejorar millones de vidas en el mundo. Por consiguiente, el gran cambio que implica implementar la sostenibilidad se basa en ser activos, tanto diseñadores y/o empresarios como consumidores y sociedad en general, así de esta manera evolucionar hacia una moda renovable y que esté conectada con el entorno ambiental y la comunidad con base en las técnicas de reutilización, transformación y reciclaje de las prendas usadas y que terminan en vertederos de basura diariamente.

La inquietud de las problemáticas asociadas a las temáticas de moda, diseño y sostenibilidad, y sus relaciones están inspiradas en el siguiente evento: el 24 de abril de 2013, en la ciudad de Dhaka, la capital de Bangladesh, un edificio de ocho pisos colapsa alrededor de las 9 a.m., donde mueren miles de personas y otras resultan heridas por el incendio del edificio llamado Rana Plaza, cuyo dueño es Sohel Rana, miembro del partido centro izquierdista Liga Awami de Bangladesh. Dentro del edificio se encontraban factorías, entidades bancarias y otras tiendas. Aunque la estructura física del edificio estaba en malas condiciones, los supervisores declaraban que era seguro. La mayoría de las víctimas fueron mujeres.

A causa de esto se generó preocupación desde distintos frentes del sistema moda, sobre todo, los diseñadores independientes y organizaciones (Common Objective, Industrial Global Union, Clean Clothes Campaign, Global Organic Textile Standard, Textile Exchange, Greenpeace International, World Fair Trade Organization y la Fair Trade International), dedicadas al comercio justo, el medio ambiente y la seguridad laboral, quienes han creado iniciativas de concientización con respecto al bienestar de los trabajadores de las fábricas textiles y, a su vez, han visibilizado las inquietudes que existen hacia las nuevas maneras de crear y hacer moda en el mundo. Es así que en el año 2014 se celebró la revolución de la moda, una iniciativa creada desde la premisa: “Quién hace tu ropa?”, para concientizar a las empresas, casas de diseño de moda, diseñadores y a consumidores, acerca de la responsabilidad social y laboral sobre cómo el hacer la moda y comprarla son importantes para crear una sostenibilidad justa y equitativa dentro de la industria per se. Esta iniciativa nace como consecuencia del caso en Bangladesh, debido a su impacto mundial que visibilizó alrededor del mundo la inequidad y la falta de balance que existe en esta comunidad creativa y productiva.

En mayo de 2013, la Escuela de negocios de Copenhague, en colaboración con la Growth from Knowledge, condujo un estudio global enfocado en la moda sostenible, por medio del cual se concluyó que existe una cultura de consumo de usar - tirar - comprar, estructura poco saludable que fomenta el consumo excesivo y el desperdicio; en efecto, la moda rápida es repetidamente criticada por crear un nivel de consumo que no es ni social ni ambientalmente sostenible y se necesita desafiar al modelo comercial dominante de la moda rápida, que solamente basa su negocio en grandes cantidades de colecciones nuevas a bajo precio y a bajo costo. A su vez, la creciente demanda de moda barata tiene un impacto negativo en el desempeño social y ambiental en la cadena de suministro de la moda.

Ahora bien, la misión de la revolución de la moda cree en la industria que valora a las personas, al ambiente, a la rentabilidad y a la creatividad de una manera equitativa. Su misión es traer a todos juntos para que eso pase, porque existe una necesidad de transformar y recuperar la relación entre la moda y los sistemas sociales y ecológicos, en tanto que la sociedad debe ser partícipe y liderar procesos de concientización y sensibilización con respecto a la responsabilidad social empresarial que debe ejercer el diseñador de moda como comunicador y educador, facilitador, activista y empresario en el marco de la sostenibilidad alrededor del mundo y del universo de la moda. A su vez, la misión de la campaña de la revolución de la moda es atraer a los involucrados para hacerlo posible, tanto al empresario (diseñador-productor textil) como al consumidor, ya que este último es quien no sabe realmente a quién beneficia a la hora de comprar su prenda de vestir, por ejemplo. 
En Colombia se ha adoptado la campaña de la revolución de la moda desde el año 2014, donde existe un colectivo robusto, que a partir de sus saberes alrededor de la moda ha llevado en conjunto una serie de eventos simbólicos que hacen una reflexión acerca de la necesidad de crear una moda sostenible. A pesar de los esfuerzos mancomunados, nosotros como Institución universitaria y desde el programa de Diseño de Modas, vemos como diseñadores la necesidad de tomar acciones en la medida en que ellas puedan ser un canalizador de nuevas creaciones a partir de la reutilización, transformación y reciclaje del material textil; en efecto, los estudiantes del programa ven con una urgente necesidad crear una marca sostenible a partir de esas tres formas de cambiar la manera en la que los seres humanos tratan los residuos textiles en la actualidad y que en realidad no se ha podido explorar en gran magnitud dentro de las instituciones educativas. Es importante recalcar que la intención también se basa en la difusión del conocimiento alrededor de este tema y que, de esta manera, los estudiantes puedan llegar al consumidor, quien debe conocer lo que sucede con respecto a ser sostenibles y ser compradores críticos y, a su vez, responsables.

Actualmente, en Colombia, se ha llevado a cabo proyectos en diseño como el proyecto WOOCOA, el cual es liderado por un grupo de investigación de la Uniandes, que consiste en la creación de una lana vegetal compuesta por fibras de cáñamo y coco tratadas con enzimas de orellana (Universidad de los Andes, 2018), y la creación de cuero vegano a base de fique, que fue patentada por parte del empresario Alejandro Moreno, quien lidera la compañía Fiquetex. Por otro lado, en México también se han llevado a cabo dichos proyectos como la creación del cuero a partir del cactus Ilamado nopal. Estos proyectos reúnen una serie de tecnologías que van encaminadas a modificar la manera en cómo se podría construir nuevas fibras, es por esto que los residuos textiles como es la ropa de segunda mano en Colombia, no se ha explorado la reutilización, la transformación y el reciclaje de las prendas desde una construcción del diseñador de moda y es en esta premisa en donde los diseñadores del Politécnico Grancolombiano desean trabajar para así poder encontrar vías en las cuales los residuos, tanto de prendas que no se utilizan como los residuos textiles en sí, pueden llegar a transformarse en nuevas prendas y en nuevas maneras de sensibilización colectivas e individuales, y así generar una economía circular y sostenerse dentro de la industria de la moda del país.

Durante el año 2020 se han evidenciado múltiples cambios en la humanidad; la comunicación y el medio por el cual cada persona se desenvuelve se ha transformado, la virtualidad se ha vuelto vital para la socialización y se ha normalizado como método de aprendizaje para niños, jóvenes y adultos. Será posible evidenciar que a nivel mundial el impacto económico afectará las personas, y a su vez a las cadenas de valor y los mercados. El de indumentaria será una de ellas. Suárez (2020) afirma que: "Según el Informe del sector moda en España publicado por EY, en 2020 se estima una caída de ingresos de hasta el 35-40\%, esto supone una pérdida muy grande de entre unos seis mil y siete mil millones de euros", no obstante, este tiempo permitió generar estrategias de venta y tratar de recuperar una parte de las inversiones realizadas, incentivando las ventas online de manera exhaustiva. Las pérdidas dieron paso a la experimentación a fondo en otros campos. Debido a la pandemia que habita en el mundo, las tiendas físicas han pasado a un segundo plano para dar paso a las ventas en plataformas digitales "vamos a evolucionar hacia lo que todos saben que es una especie de nueva normalidad" (JLL, 2020), señaló Pete Nordstrom en un panel organizado por Vogue.

\section{MÉTODO}

La heurística que se ha manejado en este proyecto de investigación-creación ha estado enfocada en un primer acercamiento hacia la búsqueda de fuentes escritas que hacen referencia a temas relacionados con la sostenibilidad en la moda desde diferentes perspectivas y/o labores del diseñador como: comunicador, educador y facilitador para el desarrollo de un estado del arte para crear un artículo de revisión acerca del tema sostenible en Colombia y en el mundo. A su vez, la metodología propuesta para el desarrollo de este proyecto está basada en el Design Thinking (diseño de pensamiento), el cual describe los pasos que el diseñador emplea al momento 
de crear y proyectar; de igual manera, tiene el propósito de conocer los problemas, necesidades o deseos de los usuarios y ofrecer soluciones.

Ahora bien, dentro del proceso del proyecto se van a desarrollar cursos acerca de reutilización, transformación y reciclaje de prendas con los estudiantes de diseño de modas del Politécnico Grancolombiano y convertirlos en talleristas de la Institución, para así crear talleres de sensibilización dentro de la planta de confección de la universidad y generar productos con base en prendas de segunda mano, ya sean donadas o traídas. De esta manera, utilizar la plataforma de Instagram para difundir tutoriales con respecto a la sostenibilidad en términos conceptuales y también cómo crear prendas a partir de los talleres realizados. De igual manera, la plataforma YouTube funcionaría como un repositorio de dichos tutoriales.

\section{Metodología}

\begin{tabular}{|c|c|c|}
\hline Fase 1 & Fase 2 & Fase 3 \\
\hline $\begin{array}{l}\text { - Buscar a través de fuentes } \\
\text { escritas, temas relacionados } \\
\text { con la sostenibilidad en la moda } \\
\text { desde diferentes perspectivas } \\
\text { y/o labores del diseñador como: } \\
\text { comunicador, educador y } \\
\text { facilitador. } \\
\text { - Interpretar dicho corpus a } \\
\text { través de la visión crítica del } \\
\text { diseñador de moda del } \\
\text { Politécnico Grancolombiano } \\
\text { por medio de un Estado del } \\
\text { Arte yasí, crear unartículo para } \\
\text { una revista indexada. }\end{array}$ & $\begin{array}{l}\text { - A partir de esa } \\
\text { interpretación de fuentes, } \\
\text { desarrollar campañas de } \\
\text { visibilización con respecto } \\
\text { de la temática de la } \\
\text { sostenibilidad. } \\
\text { - Desarrollo de cursos de } \\
\text { reutilización, } \\
\text { transformación } \\
\text { recuperación en conjunto } \\
\text { con los estudiantes de } \\
\text { diseño de modas de la } \\
\text { institución y enseñarles } \\
\text { para que se conviertan en } \\
\text { talleristas de la institución. }\end{array}$ & $\begin{array}{l}\text { - Creación de talleres de } \\
\text { sensibilización dentro de la planta de } \\
\text { confección de la universidad y generar } \\
\text { productos con base en prendas de } \\
\text { segunda mano, ya sean donadas o } \\
\text { traídas. } \\
\text { - Utilizar la plataforma como } \\
\text { Instagram para difundir tutoriales con } \\
\text { respecto a la sostenibilidad en } \\
\text { términos conceptuales y también } \\
\text { como creación de prendas a partir de } \\
\text { talleres, usando YouTube, que } \\
\text { funcionaría como un repositorio de los } \\
\text { tutoriales. }\end{array}$ \\
\hline
\end{tabular}

Tabla 1 Metodología del proyecto. Elaboración propia.

\section{RESULTADOS}

Los resultados que se han obtenido hasta el momento: recolección de una base de datos robusta con base en artículos, publicaciones y audiovisuales que hacen referencia a la temática de la sostenibilidad desde el año 2013 y lo que ha ocurrido progresivamente en el sistema moda en el mundo, Latinoamérica y Colombia. A su vez, se ha venido recopilando información de ciertas personas en el país que se encuentran involucradas con el tema y serán próximas a ser entrevistadas por las estudiantes del semillero. A comienzos de diciembre del año 2020, gracias a la recolección de datos, se inició la escritura de un breve estado del arte para crear un artículo de revisión y poder publicarlo en una revista de divulgación y/o indexada. Se pudo encontrar que hay bastante información con respecto al tema de la sostenibilidad en la moda, y que puede ofrecer una cantidad considerable de contenido para la creación de artículos de investigación y/o para crear cápsulas de conocimiento en las redes sociales con las cuales el semillero contará durante el año 2021 en adelante.

De la misma manera, el grupo semillero de investigación Moda, Comunicación y Sostenibilidad, fue ganador de la convocatoria de cofinanciación de la Institución Universitaria Politécnico Grancolombiano 2020-2021, y participó el día 29 de octubre del 2020 en el Primer encuentro de investigación-creación de la Universidad del Bosque, dentro del marco del XXVI Congreso Institucional de investigaciones, con un póster digital, el cual será publicado en las redes sociales del semillero. 
Ramos, Puentes, Díaz, Ávila, Rueda, Caicedo, León, Garagoa \& Guevara

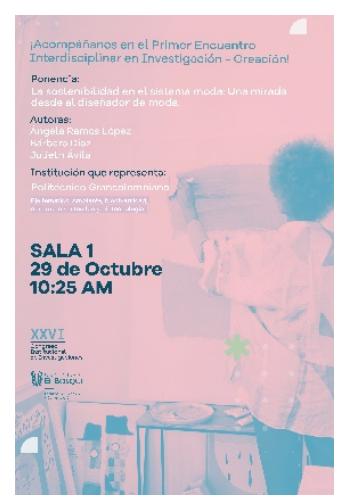

Ilustración 1. Afiche de invitación para presentación de poster digital. Imagen: Universidad del Bosque.

Ilustración 2. Imagen: Semillero Sostenibilidad

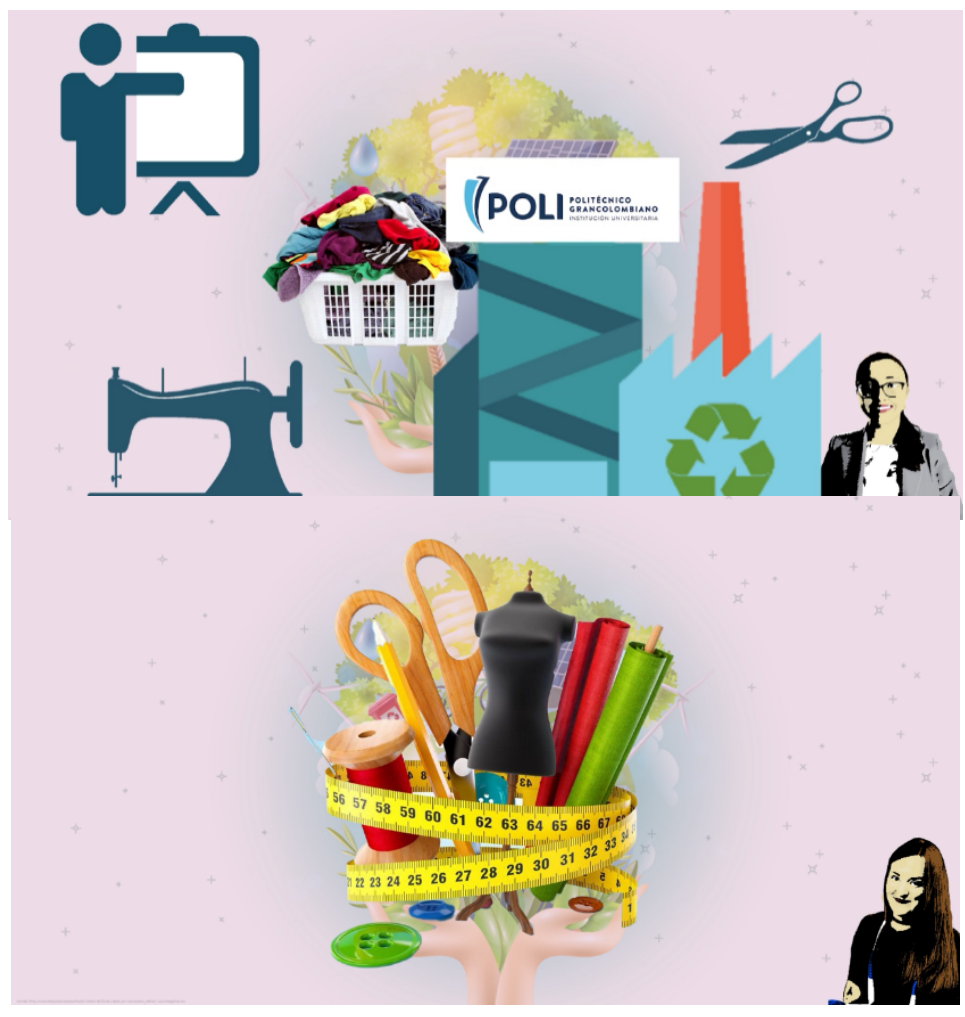

Fotograma Póster digital. Moda, Comunicación y (Policosmo). 
A su vez, el semillero participó en el Tercer encuentro Nacional de investigación de la Institución Universitaria Politécnico Grancolombiano, en donde esta ponencia tuvo una muy buena acogida por parte de los jurados y del público en general; gracias a ello se da a conocer este working paper mediante la publicación seriada llamada Encuentros con Semilleros: Aportes y reflexiones con ISSN 2711-4414 (En línea). Igualmente, la universidad dio a conocer en su portal web el proyecto del semillero en el siguiente link: https://www.poli.edu.co/noticias/semillero-de-diseno-de-modas-un-si-la-moda-sostenible el cual ha sido compartido por varios medios alternativos y redes sociales.

\section{DISCUSIÓN Y CONCLUSIÓN}

\section{Social y laboral}

El comercio justo como premisa de la equidad y el derecho al trabajo digno citado en la Carta de los Derechos humanos, como lo cita en el artículo 23, "enunciará detalladamente el derecho de toda persona al trabajo, con igual salario por trabajo igual y sin discriminación.", y "exige políticas de comercio justo para garantizar la dignidad humana".

\section{Reciclaje}

Las 5 R (rechazar, reducir, reciclar, reusar y reincorporar), como acto cooperativo de las comunidades que permitan influenciar dicha cultura y conciencia alrededor del consumo racional de prendas y productos. Es así como estas prácticas ayudan a utilizar los residuos y detienen sus efectos negativos a corto plazo, y transmiten confianza a la hora de desarrollar ideas de sostenibilidad. No obstante, son procesos en los cuales aún se necesita mucho trabajo en cuanto a la industria a mayor escala, pero que, a pesar de ello, si se desarrollan pequeños procesos de sensibilización al respecto pueden ser procesos que a futuro puedan impedir que los residuos lleguen a los vertederos de manera descontrolada, como sucede en la actualidad.

\section{ACV}

La implementación de políticas de responsabilidad de producción y fin de ciclo de vida a los productores, donde ellos apoyarán las dinámicas de ciclo cerrado en los productos una vez finalice su ciclo. De esta manera se puede generar conciencia en los consumidores, quienes pueden conocer el "metabolismo" de su armario y tener la capacidad de ajustarlo, para convertirse en lugares de "equilibrio dinámico en el cual, en este caso, la ropa se cambia, se comparte y se reutiliza sin necesitar constantemente productos y recursos nuevos" (Fletcher \& Grose, 2012, p. 88).

\section{Producción}

El fomento a la investigación asociada a procesos de producción limpia, biomateriales y diseño responsable en los que se involucre toda la cadena productiva para hacerla más consciente del uso de recursos y energía.

\section{REFERENCIAS BIBLIOGRÁFICAS}

Baptiste, B. (2020). Transformar el mundo, seis ideas para repensar la sostenibilidad. Recuperado de: http://especiales.revistaarcadia.com/libro-transformar-el-mundo-por-brigitte-baptiste/

BBC News. (2017). Rana Plaza collapse: Sohel Rana jailed for corruption. Recuperado de: https://www.bbc.com/news/world-asia-41082448

Common Objective. (s.f.). Our Story. Recuperado de: https://www.commonobjective.co/our-story 
Cruz, R. (2013). Responsabilidad Social Empresarial (RSE). Recuperado el 16 de junio de 2020, de Escuela de Organización Industrial [EOI]: https://www.eoi.es/blogs/mintecon/2013/05/28/responsabilidad-socialempresarial-rse-3l

Daza-Orozco, CE. (2014). La alienación viste a la moda: análisis fílmico desde la tipificación ideal weberiana. El ojo que piensa - Revista de cine iberoamericano, 1(9). https://doi.org/10.13140/RG.2.2.21231.66726

Ethical Fashion Forum. (2020). Ethical Fashion Forum History. Recuperado de: https://the.ethicalfashionforum.com/history

Fair Labor Association. (2012). Fair Labor Association. Recuperado de: https://www.fairlabor.org/

Fairtrade International. (s.f.). Fairtrade International. Recuperado de: https://www.fairtrade.net/

Fletcher, K., \& Grose, L. (2012). Gestionar la sostenibilidad en la moda. Diseñar para cambiar. Materiales, procesos, distribución y consumo. Barcelona: Editorial Blume.

Gwilt, A. (2014). Moda sostenible, manuales de diseño de moda. Barcelona: Editorial Gustavo Gilli.

Howarth, G., \& Hadfield, M. (2006). A sustainable product design model. Materials and Design, (27), 1128-1133.

Semana Sostenible. (2020). Hábitos de consumo y acciones favorables. Recuperado de: https://sostenibilidad.semana.com/negocios-verdes/multimedia/habitos-consumo-colombianos/33539

Universidad EAN. (2020). Moda sostenible, una mirada al presente y futuro de la moda [Webinar]. Recuperado de: https://universidadean.edu.co/eventos/webinar-moda-sostenible-una-mirada-al-presente-y-futuro-de-lamoda

Vega, C. (2020). Colombia tendrá fábrica de cuero vegano. Recuperado de: https://www.elespectador.com/noticias/economia/colombia-tendra-fabrica-de-cuero-vegano/ 